\title{
Selection of the Optimum Electrospray Voltage for Gradient Elution LC-MS Measurements
}

\author{
Ioan Marginean, Ryan T. Kelly, Ronald J. Moore, David C. Prior, \\ Brian L. LaMarche, Keqi Tang, and Richard D. Smith \\ Biological Sciences Division, Pacific Northwest National Laboratory, Richland, Washington, USA
}

\begin{abstract}
Changes in liquid composition during gradient elution liquid chromatography (LC) coupled to mass spectrometry (MS) analyses affect the electrospray operation. To establish methodologies for judicious selection of the electrospray voltage, we monitored in real time the effect of the LC gradient on the spray current. The optimum range of the electrospray voltage decreased as the concentration of organic solvent in the eluent increased during reversed-phase LC analyses. These results and related observations provided the means to rationally select the voltage to ensure effective electrospray operation throughout gradient-elution LC separations. For analyses in which the electrospray was operated at constant voltage, a small run-to-run variation in the spray current was observed, indicating a changing electric field resulting from fouling or degradation of the emitter. Algorithms using feedback from spray current measurements that can maintain the electrospray voltage within the optimum operating range throughout gradient elution LC-MS were evaluated. The electrospray operation with voltage regulation and at a constant, judiciously selected voltage during gradient elution LC-MS measurements produced data with similar reproducibility. (J Am Soc Mass Spectrom 2009, 20, 682-688) (c) 2009 Published by Elsevier Inc. on behalf of American Society for Mass Spectrometry
\end{abstract}

$\mathrm{E}$ lectrospray ionization (ESI) has enabled the successful routine on-line coupling of liquid chromatography (LC) with mass spectrometry (MS) [1, 2], a combination that has become the de facto standard for proteomics research [3, 4]. Despite the tremendous technological growth of LC-MS, the electrospray remains a seemingly robust but relatively neglected and poorly understood intermediate. It is expected that shifting eluent composition during gradient LC separations may alter the electrospray operation; however, essentially all gradient-elution LC-MS analyses are performed with little or no feedback with respect to electrospray performance.

During LC-MS analyses, the solvent composition and flow rate are dictated by the separation conditions and generally only the applied voltage and the emitter-MS inlet distance can be adjusted. Direct optimization of the analyte signal before data acquisition is not possible, as for infusion experiments. The voltage is usually selected according to the instrument manufacturer's recommendations or previous experience, rather than careful consideration of the experimental conditions. Emitter-MS inlet distance adjustments induce large changes in the electric field driving the electrospray because of the nonlinear dependence between the two parameters. This may result in different electrospray current or even a different operating regime [5]. When

Address reprint requests to Dr. Richard D. Smith, Pacific Northwest National Laboratory, Biological Systems Analysis and Mass Spectrometry, 3335 Q. Avenue (K8-98), P.O. Box 999, Richland, WA 99352. E-mail: rds@ pnl.gov nonmetallic (e.g., fused silica) emitters are used, the voltage is usually applied upstream of the emitter via a conductive material in contact with the solution. The voltage drop across the emitter should also be considered in this case [6]; applying the same voltage on emitters of different lengths positioned at the same distance in front of the MS inlet would also result in different electric fields.

The voltage that ensures optimum operation at the beginning of the analysis may not be appropriate for the solvent mixture eluting at the end of the gradient. In previous work, the electrospray characteristic curves measured with several solvent compositions simulating a typical reversed-phase liquid chromatography (RPLC) gradient indicated that the voltage required to maintain the electrospray in a specific operating regime decreases significantly with increasing organic solvent concentration [5]. The vast majority of LC-MS analyses are currently performed with constant electrospray voltage; previous experience with a specific type of analysis may help in guiding the selection of a reasonable voltage for the beginning of the gradient that is not too high or too low for the end of the gradient. The changing eluent composition can be counterbalanced by post-column combination with an inverted gradient [7]; this approach provides steady solvent composition throughout the analysis at the cost of decreased sensitivity because of analyte dilution and increased flow rate.

Only a few attempts to rationally control the electrospray operation during LC-MS analyses have been described. Valaskovic et al. [8] used feedback from an electrospray imaging system to automatically adjust the
(C) 2009 Published by Elsevier Inc. on behalf of American Society for Mass Spectrometry. 1044-0305/09/\$32.00

doi:10.1016/j.jasms.2008.12.004
Published online December 13, 2008 Received September 23, 2008 Revised December 3, 2008 Accepted December 7, 2008 
applied voltage and control its operating regime. Two recently reported techniques aimed at developing feedback mechanisms based on spray current measurements [9, 10]. Staats et al. [9] sought to account for sudden interruptions of the spray arising from emitter clogging, air bubbles, or changes in the eluent composition by adjusting the distance between the emitter and the MS inlet in response to changes in spray current. Gapeev et al. [10] independently built a feedback circuit very similar to the one we described [11] and used it to maintain the electrospray current at a certain level by adjusting the applied voltage. Both techniques disregarded the potential for electrospray switching between operating regimes.

The present report provides insights into the electrospray operation during LC-MS analyses. It is shown that changes in eluent composition during a gradient RPLC analysis are reflected by shifting electrospray characteristic curves. A slow drift of the current generated by an electrospray operated at constant voltage is also demonstrated between LC runs. Two approaches to control the applied voltage through feedback from spray current measurements are discussed.

\section{Experimental}

\section{Sample Preparation}

Acetic acid (HOAc), trifluoroacetic acid (TFA), and acetonitrile were purchased from Sigma-Aldrich (St. Louis, MO, USA), and water was purified using a Barnstead Nanopure Infinity system (Dubuque, IA, USA). Solvents A and B consisted of $0.2 \%$ HOAc + $0.05 \%$ TFA in water and 0.1\% TFA in 90:10 acetonitrile: water, respectively. The two solvents were degassed before use by vacuum filtration followed by helium sparging for $10 \mathrm{~min}$. Proteolytic digestion of bovine serum albumin (BSA; Pierce Biotechnology, Rockford, IL, USA) was conducted using sequencing grade trypsin (Promega, Madison, WI, USA) according to established procedures [12]. The BSA tryptic digest was diluted to a concentration of $0.1 \mu \mathrm{g} / \mu \mathrm{L}$ in purified water and the stock solutions were refrigerated for later use.

\section{Reversed-Phase Liquid Chromatography}

LC systems similar to those used here have previously been described in detail $[13,14]$. Briefly, solvents A and B were loaded into a pair of 100-mL Isco 100DM syringe pumps controlled by a series D controller (Isco, Lincoln, NE, USA). A two-position, four-port manually operated valve (Valco Instruments Co., Houston, TX) selected one of the solvents to be delivered into a $2.5-\mathrm{mL}$ mobile phase dynamic mixer (fabricated in-house). The samples were injected using a two-position, six-port valve (Valco Instruments Co.) with a 5- $\mu \mathrm{L}$ loop. The reversedphase capillary HPLC column was prepared in-house by slurry packing $3-\mu \mathrm{m}$ Jupiter C18 stationary phase
(Phenomenex, Torrence, CA, USA) into a 60-cm-long, 360- $\mu \mathrm{m}$ o.d. $\times 75-\mu \mathrm{m}$ i.d. fused silica capillary tubing (Polymicro Technologies Inc., Phoenix, AZ, USA) incorporating a $0.5-\mu \mathrm{m}$ retaining screen in a $1 / 16$-in. union (Valco Instruments Co.) custom bored to $75 \mu \mathrm{m}$ i.d. (Lenox Laser, Glen Arm, MD, USA). When equilibrated at 5000 psi with $100 \%$ mobile phase A, the HPLC system delivered a flow of close to $20 \mu \mathrm{L} / \mathrm{min}$, which was split with a 30-cm-long, 20- $\mu \mathrm{m}$-i.d. fused silica tubing to provide about $200 \mathrm{~nL} / \mathrm{min}$ flow through the HPLC column. Following a 60-min column regeneration with solvent $A$ and 30-min sample load time, the solvent selection valve was switched to deliver solvent B into the mixer, thus creating an exponential gradient.

\section{Spray Current Measurements}

A schematic of the instrument setup is presented in Figure 1. The solution eluting from the chromatographic column was delivered through $20-\mu \mathrm{m}$-i.d. electrospray emitters prepared by chemically etching fused silica capillaries [15]. High voltage generated by a Bertan 205B-03R (Hicksville, NY, USA) power supply was applied through a previously described ammeter [11] to the stainless steel union holding the emitter. In this configuration the ammeter measures both the current delivered by electrospray and the current leaking to ground through the HPLC system. To minimize the contribution of the current leaking through the LC column on the spray current measurements, the unions at the two ends of the column were maintained at similar potentials. A USB-6251 Multifunction Data Acquisition board (National Instruments, Austin, TX, USA) was used to monitor the ammeter output and to remotely control the high-voltage power supply.

\section{MS Measurements}

Mass spectra were collected using a Micromass Q-TOF Ultima mass spectrometer (Waters Corporation, Milford, MA, USA) with the standard interface replaced by an ion funnel interface with a heated capillary inlet. Decon2LS [16] and MultiAlign [17], open-source pro-

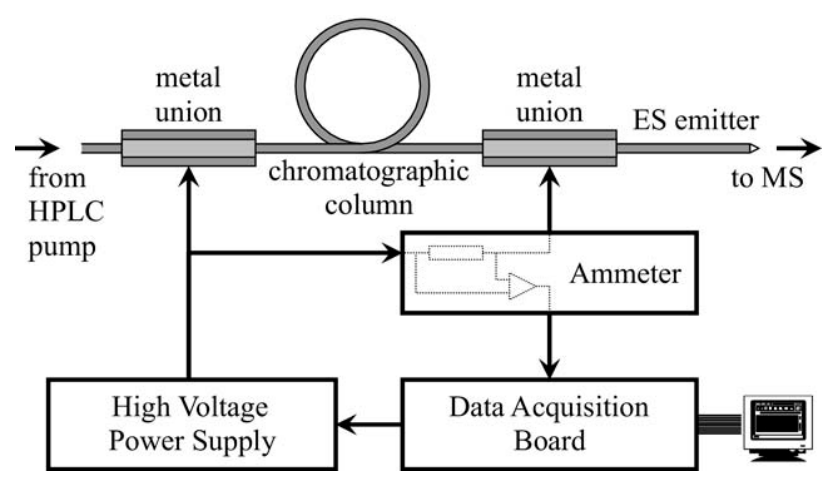

Figure 1. Instrument setup. See Marginean et al. [11] for a detailed description of the ammeter circuit. 
grams developed at PNNL and freely available at http://omics.pnnl.gov, were used for data deconvolution and alignment, respectively.

\section{Results and Discussion}

The present study uses a constant-pressure LC system delivering a flow of about $200 \mathrm{~nL} / \mathrm{min}$, which is amenable to high-throughput, automated LC-MS analyses [18]. At this flow rate, the electrospray characteristic curve (see Figure 1A in Marginean et al. [5]) can be divided into three sections, each approximating an ohmic response to the applied voltage. At relatively low voltages the liquid wets the outer wall of the emitter; the current increases and the wetted area decreases with increasing applied voltage. The slope decreases slightly when the liquid anchors to the emitter rim; this point will be referred to as the "anchoring point." The second section of the curve resembles the pulsating regime observed at lower flow rates but does not have the same self-regulating character. A corona discharge most likely contributes to the current at larger applied voltages [11], when another change of slope is visible in the characteristic curve; the corresponding point will be referred to as the "discharge point." The cone-jet regime could not be established under these experimental conditions. The emitter length and/or the distance to the MS inlet change the onset voltage of the characteristic curve, but not its appearance.

The appearance of the characteristic curve can be significantly altered by emitter aging, an unavoidable process accompanied by visual and operational clues $[19,20]$. The walls of fused silica emitters-initially transparent-turn translucent; sometimes residue deposits become visible. Geromanos et al. [21] reported cavitation and/or cracking of the emitters operated at excessive field strengths. Emitter aging is generally a slow process (days), but can be accelerated significantly (hours) by operating the electrospray at voltages above the discharge point. Electrospray operation at voltages below the anchoring point can also promote the deposition of residue on the emitter outer wall; however, the emitter aging kinetics seems to be less affected. These physical and/or chemical processes eventually change the wetting properties of the emitter; the liquid maintains the contact with the outer emitter wall even at voltages above the anchoring point, swinging the electrospray to an alternative path in the parameter space. The new path is characterized by poorer reproducibility and noisier current measurements (not shown) because of more erratic fluid dynamics at the tip of a wetted emitter. During this study we tried to prolong the emitter lifetime by avoiding the electrospray operation at voltages above the discharge point. With a single exception (see Figure 2), we also maintained the voltage above the anchoring point.

The characteristic curves obtained for a blank RPLC run, shown in Figure 2 in a color-coded representation, form a gradient map. A base-peak chromatogram, col-

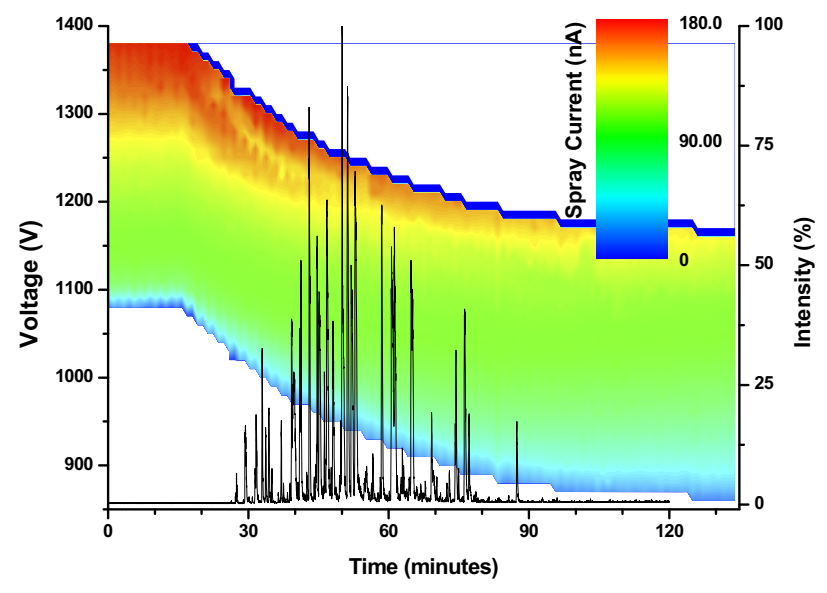

Figure 2. Electrospray characteristic curves measured during a gradient LC-MS dry run superimposed on a base peak ion chromatogram to illustrate the domain of analytical interest.

lected at constant voltage, is also provided to illustrate the gradient region of analytical interest. The voltage was scanned in steps of $10 \mathrm{~V}$ over a $300-\mathrm{V}$ range above the value generating a threshold spray current, which was set at $30 \mathrm{nA}$ to ensure uninterrupted electrospray operation. During the first $100 \mathrm{~min}$ of the gradient delivered by the constant-pressure LC system, the flow rate increases from about 200 to $250 \mathrm{~nL} / \mathrm{min}$ because of decreasing eluent viscosity. The gradient map for an LC system operated at constant flow $(\sim 200 \mathrm{~nL} / \mathrm{min})$ is expected to be slightly different from that in Figure 2. The spray current would be similar at the beginning of the gradient, but would shift to roughly $10 \%$ smaller values at the end of the analysis. The onset voltages are expected to be insignificantly shifted toward lower values; however, the discharge voltages are somewhat smaller at 200 than at $250 \mathrm{~nL} / \mathrm{min}$ [5]. Along with an expected slight decrease in ion utilization efficiency, the increased flow rate is expected to improve the electrospray robustness.

During the time required for the liquid to travel from the mixer to the emitter tip ( $\sim 15 \mathrm{~min})$ no change in the characteristic curves was observed. A significant drop in the threshold voltage (from 1080 to $870 \mathrm{~V}$ ) is readily visible after $100 \mathrm{~min}$. This is related to the decreasing surface tension of the eluent as the concentration of the organic component increases. A voltage set $300 \mathrm{~V}$ above the threshold generated close to $175 \mathrm{nA}$ at the beginning of the gradient and only about $135 \mathrm{nA}$ at the end of the analysis. The decrease of the characteristic curve slope is a result of the smaller eluent conductivity. Several minutes after the beginning of the gradient reached the emitter tip, the characteristic curves deviated from the theoretical shape discussed earlier. The linear dependence between the spray current and the applied voltage was disrupted by the electrospray switching to a multi-jet (rim emission) regime at the top of the applied voltage domain. This regime change produced the yellow peninsula (current $\sim 135 \mathrm{nA}$ ) in the gradient map. 
Given the changing eluent properties during the LC gradient, operating the electrospray at constant voltage can be problematic. For example, a voltage that delivers abundant current at the beginning of the gradient could later induce a corona discharge regime. Another scenario could follow the horizontal line corresponding to an applied voltage of about $1275 \mathrm{~V}$, which ensured a current of close to $135 \mathrm{nA}$ at the beginning of the gradient. The current would initially increase as the gradient reaches the emitter tip, then decrease as a result of the electrospray regime shift, and then increase again with increasing content of organic solvent in the eluent. Because of decreasing eluent conductivity, the current would finally decrease by the end of the gradient. Performing the analysis at a voltage that ensures a current $<135 \mathrm{nA}$ at the end of the gradient $(\sim 1150 \mathrm{~V}$ in this case) seems to be the safest choice. A voltage above the anchoring point at the beginning of the gradient that remains below the corona discharge point at the end of the gradient would be ideal for RPLC-MS analyses conducted with constant electrospray voltage.

Characteristic curves measured at increasing/decreasing emitter-MS inlet distance show voltage shifts to larger/smaller values. Similarly, shorter/longer emitters shift the voltage range to smaller/larger values (not shown). These conditions were maintained during each set of measurements presented (see Figures 3 and 5); however, a new emitter was used for each set of measurements. It is thus likely that the emitters did not have exactly the same length and/or were positioned at slightly different distance from the MS inlet. Consequently, voltage values are strictly comparable only within the same set of measurements and thus voltage offsets between sets of measurements are not surprising. The current delivered by the electrospray is a better indicator of the electric field established at the emitter tip and is thus a better metric to compare different sets of measurements.

Figure $3 a$ presents the base peak ion chromatogram and the spray current measured during an RPLC-MS analysis performed with the electrospray voltage set at $1250 \mathrm{~V}$. The voltage was selected to maintain a spray current $<140 \mathrm{nA}$ throughout the gradient. In terms of current generated by the electrospray, these operating conditions would be equivalent to an applied voltage of close to $1200 \mathrm{~V}$ in Figure 2. The first $60 \mathrm{~min}$ of the spray current trace correspond to the column regeneration phase. The arrow marks the sample injection time; the introduction of the sample loop into the circuit led to a slightly smaller spray current. The spray current decreased significantly when the solvent from the sample injection loop reached the emitter $(\sim 13 \mathrm{~min}$ later) because of the low solution conductivity. The gradient was initiated at time 0 .

Figure $3 \mathrm{~b}$ shows spray current traces corresponding to four consecutive RPLC-MS analyses performed as described earlier. Run-to-run variations are visible in this representation, especially for the black trace. We suspect that residue accumulation on the emitter, which
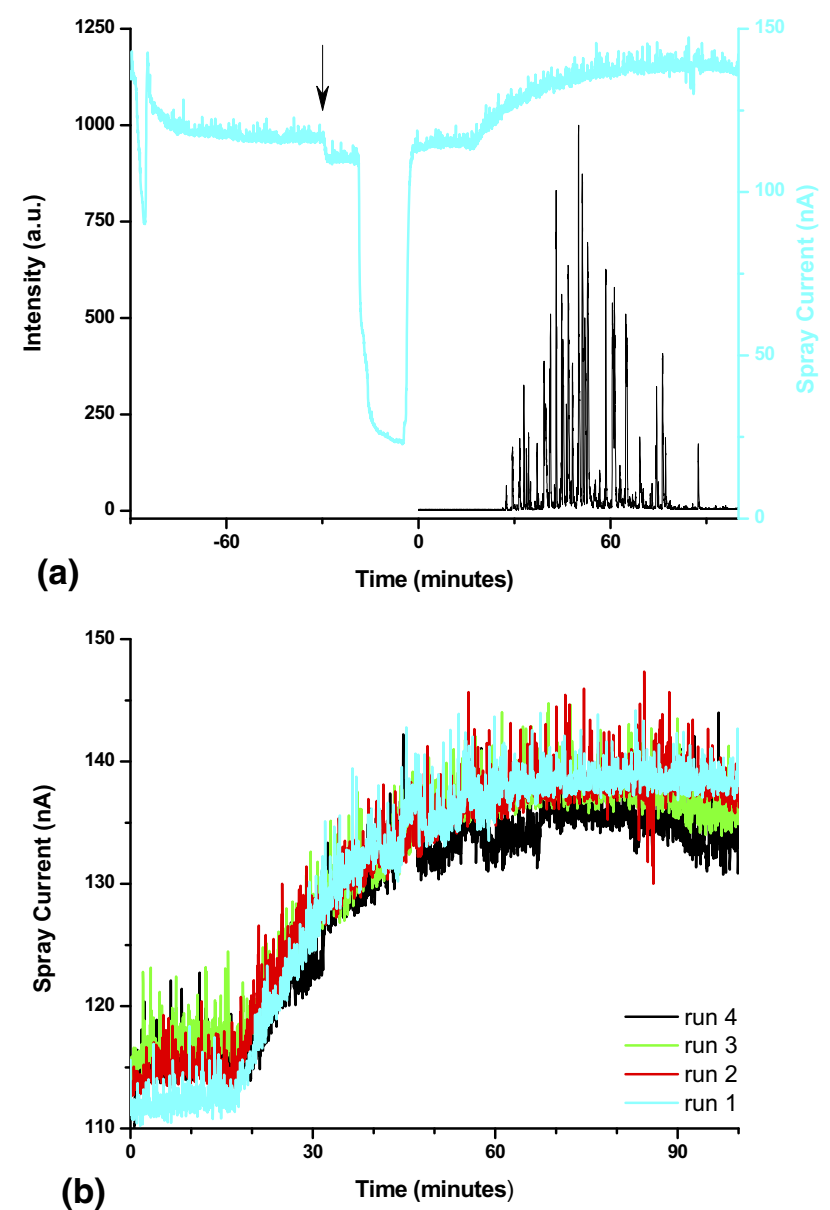

Figure 3. Base peak ion chromatogram and spray current for LC-MS analysis performed with constant $(1250 \mathrm{~V})$ electrospray voltage (a). Spray current traces for four consecutive LC-MS analyses (b).

changes the rim geometry and alters the electric field driving the electrospray, is responsible for this drift. Earlier results [22] also indicated spray current decays during long-term electrospray operation at constant voltage as well as the need to increase the voltage for maintaining a specific operating regime.

The spray current measurements can be used as feedback to actively maintain the electrospray between the anchoring and the corona discharge points. The corresponding section of the characteristic curve is fairly linear and can be characterized by two coefficients: the slope and the threshold voltage $\left(V_{t}\right)$. These parameters can be calculated by linear regression at any point of the gradient by changing the voltage within a limited range and measuring the corresponding current.

Considering the change in slope at the anchoring point, clearly no theoretical significance can be associated with $V_{t}$; the practical threshold voltage ensuring the delivery of minimum current by the electrospray is always larger than $V_{t}$ calculated as just described. The voltage range scanned to collect the data necessary to calculate $V_{t}$ should be as small as possible to minimize 


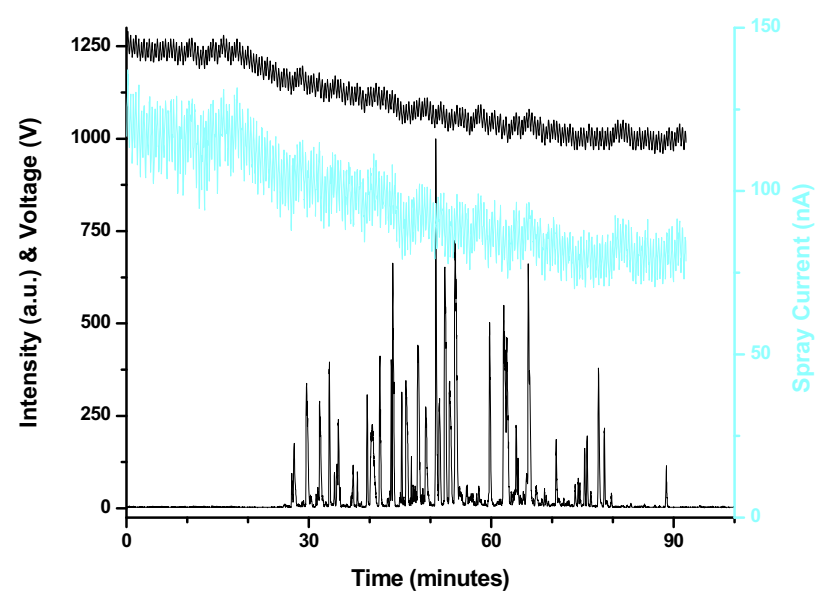

Figure 4. LC-MS analysis performed using feedback from spray current measurements to control the voltage with minimal a priori knowledge about the LC gradient (see text for details).

spray current variation that may affect the MS signal. Also, the voltage should be considerably larger than $V_{t}$ to maintain a reasonable spray current throughout the analysis. Consequently, the extrapolation used to calculate $V_{t}$ is prone to relatively large errors. However, based on $V_{t}$ values calculated along the gradient, the applied voltage may be adjusted to maintain the electrospray at equivalent points on the continuously shifting characteristic curves. For example, Figure 4 summarizes the results of an LC-MS run using this feedback algorithm. During this experiment, $V_{t}$ values were calculated by scanning the voltage in $10-\mathrm{V}$ steps within a $50-\mathrm{V}$ range and the limits of the voltage range were continuously adjusted to maintain the electrospray at least $400 \mathrm{~V}$ above $V_{t}$.

The voltage decreased from an average of approximately $1250 \mathrm{~V}$ at the beginning of the gradient to an average of about $1000 \mathrm{~V}$ at the end of the gradient. Decreasing eluent conductivity led to the spray current declining from an average of about 120 to $80 \mathrm{nA}$ during the analysis. The voltages generating similar currents in Figure 2 were about 1230 and $1010 \mathrm{~V}$, respectively. This approach leads to relatively large spray current fluctuations, which could be minimized if the determination of new $V_{t}$ values would be triggered only by significant changes in the spray current or regular time intervals.

The feedback algorithm easiest to implement adjusts the applied voltage to maintain a given spray current. In 1972, Evans and Hendricks [23] built a feedback circuit that regulated the voltage to avoid spontaneous changes in the emission of a liquid metal ion source. Gapeev et al. [10] implemented a similar approach using a proportional-integral-derivative (PID) algorithm, but did not provide any guidance on selecting the spray current level to be maintained. The results presented in this report suggest a linear behavior of the spray current as a function of the applied voltage for our particular experimental conditions. This system should be regulated effectively by a PID algorithm; however, the electrospray characteristic curves are not always linear [5, 24]. Even if the assumption of linearity were correct, air bubbles-which are common in LC systems-induce significant spray current drops, which would lead to large voltage overshoots.

Figure 2 suggests that spray currents as high as 135 $n A$ could be safely maintained throughout the analysis; different solvent combinations or flow rates would require a re-evaluation of this value. Figure 5a summarizes the results of a typical RPLC-MS analysis in which the voltage was continuously adjusted to maintain the current at a conservative level of $120 \mathrm{nA}$ throughout the experiment. As in Figure 3a, the first 60 min of the spray current trace correspond to column regeneration. Immediately after sample injection (marked by an arrow), the voltage increased to compensate for the small current drop, after which it was maintained constant during elution of the low-conductivity sample solvent. The gradient was also initiated at time 0 .

Figure $5 \mathrm{~b}$ presents the voltage applied during four consecutive RPLC-MS runs. In good agreement with expectations from Figure 2 (a voltage drop from 1230 to $1130 \mathrm{~V}$ ), the voltage dropped roughly $100 \mathrm{~V}$ by the end
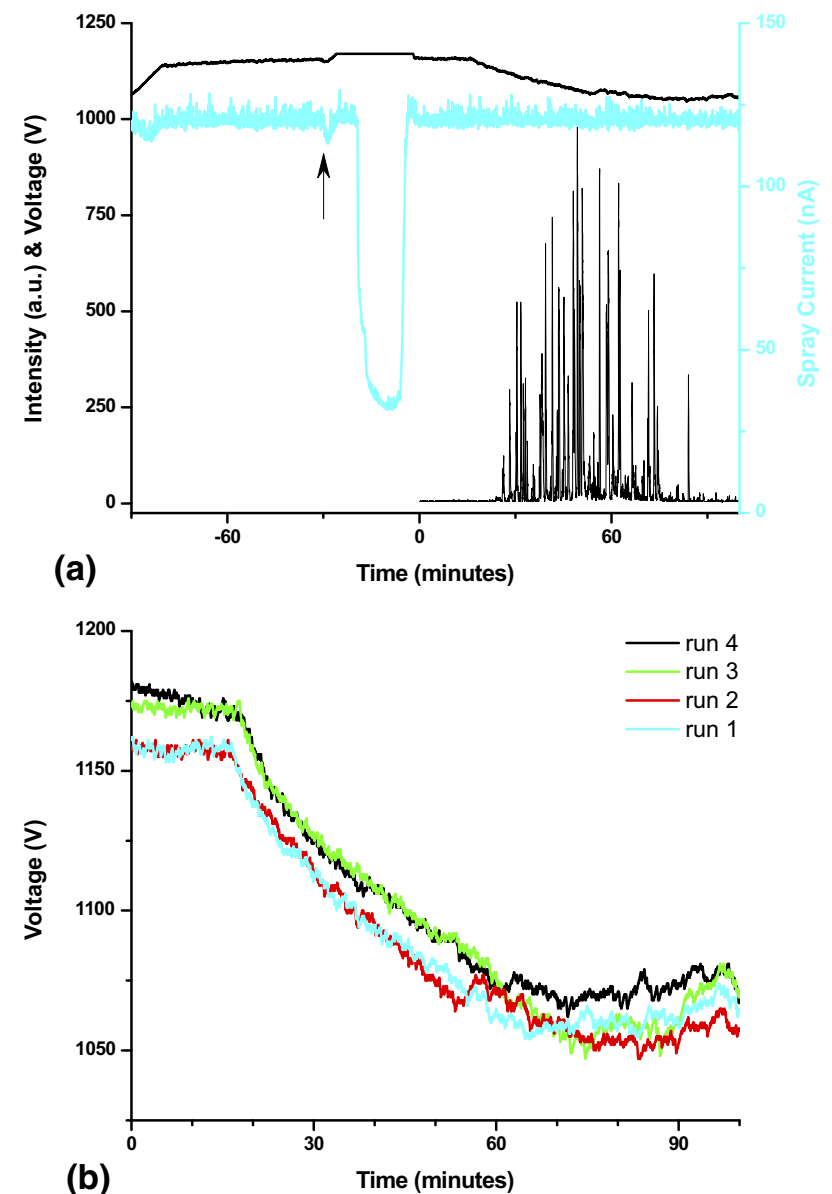

Figure 5. Base peak ion chromatogram, applied voltage and spray current for LC-MS analysis performed with feedback control of the applied voltage (a). Applied voltage traces for four consecutive LC-MS analyses (b). 
of the gradient (from 1160 to $1060 \mathrm{~V}$ ). This voltage drop was smaller than that observed with the previous algorithm $(\sim 250 \mathrm{~V})$ and may seem minor when compared to an applied voltage of close to $1200 \mathrm{~V}$; however, the voltage drop is significant in comparison with the voltage range that affords the electrospray operation between the anchoring and the corona discharge points.

To maintain a current of $120 \mathrm{nA}$, the voltage increased slightly at the end of the gradient, compensating for the lower eluent conductivity. There was no major difference between the voltage traces during the first two runs, but the voltage increased nearly $30 \mathrm{~V}$ to generate the same current during the last two runs. As in the case of the experiments performed at constant voltage, we suspect that microscopic deposits on the emitter modified its rim structure, affecting the electric field at the tip. Running the same set of measurements at constant voltage would result in slightly lower spray currents during the last two runs than during the first two runs.

There is a significant difference between the voltage values (1230 and $1160 \mathrm{~V}$ ) that ensured the generation of similar spray current (120 nA) in Figures 2 and 5; this may be related to different emitter length and/or positioning relative to the MS inlet. Assuming that operation at constant voltage was desirable for the experiments in Figure 5, a voltage of $1230 \mathrm{~V}$ would be selected using Figure 2 as benchmark and without any hint of this voltage shift. The resulting electric field would draw about $155 \mathrm{nA}$ at the beginning of the analysis, which would be appropriate at the start of the gradient but excessive at the end.

To evaluate the effect of the small run-to-run spray current drift observed when the electrospray was operated at constant voltage (Figure $3 b$ ), we considered the species detected in all four measurements to calculate the reproducibility of the corresponding analytical signal. The coefficient of variance was slightly lower than $30 \%$, a level roughly equivalent to that found for label-free quantitation in LC-MS studies $[25,26]$. The measurements with feedback control (Figure 5b) resulted in a similar coefficient of variance. It may seem surprising that compensating for the spray current drift did not improve the reproducibility of the data. We can interpret this observation in the light of our recent results [11], showing that changes in ionization and transmission efficiencies offset one another for electrosprays operated at relatively large flow rates, offering greater robustness at the cost of smaller ion utilization efficiency.

\section{Conclusions}

Spray current measurements can be used to monitor the electrospray operation throughout gradient LC-MS measurements. Even if not used as feedback for voltage control, they can still provide a reliable quality control mechanism and important information regarding electrospray operation by diagnosing emitter clogging, the presence of air bubbles in the eluent, and emitter aging. Based on gradient maps, the electrospray voltage can be rationally selected to ensure optimal operation throughout the analysis. We expect that the small current drift observed during the four LC-MS runs at constant voltage (Figure $3 b$ ) becomes more significant with emitter aging and would eventually alter the quality of the data collected at constant electrospray voltage. We are in the process of integrating the spray current monitoring and voltage selection/regulation approaches in our automated LC-MS systems [18]. This will enable longerterm studies of electrospray behavior that will further clarify the effect of voltage regulation on the LC-MS data reproducibility.

We have shown that our particular experimental conditions afford the electrospray operation at a constant but rigorously selected voltage with no negative impact on the reproducibility of the MS signal. However, these results should not be interpreted to suggest that an electrospray can be operated at constant voltage without unfavorable consequences throughout any LC-MS experiment. Selection of the electrospray voltage for the vast majority of LC-MS analyses is less rigorous than that presented here; the voltage optimization at the beginning of the analysis often results in poor electrospray operation at the end of the gradient. Changing electrospray operating regimes and/or faster emitter aging arising from wetting/corona discharges are expected to induce larger run-to-run spray current fluctuations, which could affect the analyte ionization and the MS signal more significantly.

This study is most relevant for experiments in which the electrospray characteristic curves are virtually linear. Experimental conditions that bring more definition to the shape of the characteristic curves (different solvent systems, solvents of much lower conductivity, lower flow rates, flow split through multi-emitter arrays [27]) are expected to benefit significantly from more sophisticated voltage-control algorithms.

\section{Acknowledgments}

We thank Dr. Yehia M. Ibrahim and Heather M. Mottaz for providing bovine albumin samples; Dr. Eric A. Livesay, Rui Zhao, Daniel Orton, and Dr. Kostantinos Petritis for assistance with LC-MS operation; and Dr. Christina M. Sorensen, Dr. Tyler Heibeck, Dr. Vlad A. Petyuk, and Dr. Ashoka D. Polpitiya for sharing their experience with LC-MS data analysis procedures. This research was supported by the NIH National Center for Research Resources (RR-018522). Experimental portions were performed in the Environmental Molecular Sciences Laboratory, a DOE national scientific user facility located at the PNNL in Richland, Washington. PNNL is a multiprogram national laboratory operated by Battelle for the DOE under Contract DE-AC05-76RLO 1830.

\section{References}

1. Aleksandrov, M. L.; Gall, L. N.; Krasnov, V. N.; Nikolaev, V. I.; Pavlenko, V. A.; Shkurov, V. A.; Baram, G. I.; Gracher, M. A.; Knorre, V. D.; Kusner, Y. S. Direct Coupling of a Microcolumn Liquid Chromatograph and a Mass Spectrometer. Bioorg. Khim. 1984, 10, 710-712. 
2. Whitehouse, C. M.; Dreyer, R. N.; Yamashita, M.; Fenn, J. B. Electrospray Interface for Liquid Chromatographs and Mass Spectrometers. Anal. Chem. 1985, 57, 675-679.

3. Aebersold, R.; Mann, M. Mass Spectrometry-Based Proteomics. Nature 2003, 422, 198-207.

4. Domon, B.; Aebersold, R. Mass Spectrometry and Protein Analysis. Science 2006, 312, 212-217.

5. Marginean, I.; Kelly, R. T.; Page, J. S.; Tang, K. Q.; Smith, R. D. Electrospray Characteristic Curves: In Pursuit of Improved Performance in the Nanoflow Regime. Anal. Chem. 2007, 79, 8030-8036.

6. Jackson, G. S.; Enke, C. G. Electrical Equivalence of Electrospray Ionization with Conducting and Nonconducting Needles. Anal. Chem. 1999, 71, 3777-3784

7. Ramanathan, R.; Zhong, R.; Blumenkrantz, N.; Chowdhury, S. K.; Alton, K. B. Response Normalized Liquid Chromatography Nanospray Ionization Mass Spectrometry. J. Am. Soc. Mass Spectrom. 2007, 18, 1891-1899.

8. Valaskovic, G. A.; Murphy, J. P.; Lee, M. S. Automated Orthogonal Control System for Electrospray Ionization. J. Am. Soc. Mass Spectrom. 2004, 15, 1201-1215

9. Staats, S. L. T.; Fogiel, A. J.; Suna, A. Active Spray Control with Electric Field Optimization for Online NanoLC with Polymeric Spray Tips. In Proceedings of the 56th ASMS Conference on Mass Spectrometry, Denver, CO, June 1-5, 2008

10. Gapeev, A.; Berton, A.; Fabris, D. Current-Controlled Nanospray for the Analysis of Less Than Ideal Samples. In Proceedings of the 56th ASMS Conference on Mass Spectrometry, Denver, CO, June 1-5, 2008.

11. Marginean, I.; Kelly, R. T.; Prior, D. C.; LaMarche, B. L.; Tang, K.; Smith, R. D. Analytical Characterization of the Electrospray Ion Source in the Nanoflow Regime. Anal. Chem. 2008, 80, 6573-6579.

12. Kinter, M. M.; Sherman, N. E. Protein Sequencing and Identification Using Tandem Mass Spectrometry. New York: Wiley Interscience, 2000.

13. Shen, Y. F.; Tolic, N.; Zhao, R.; Pasa-Tolic, L.; Li, L. J.; Berger, S. J.; Harkewicz, R.; Anderson, G. A.; Belov, M. E.; Smith, R. D. HighThroughput Proteomics Using High Efficiency Multiple-Capillary Liquid Chromatography with On-line High-Performance ESI FTICR Mass Spectrometry. Anal. Chem. 2001, 73, 3011-3021.

14. Shen, Y. F.; Zhao, R.; Belov, M. E.; Conrads, T. P.; Anderson, G. A.; Tang, K. Q.; Pasa-Tolic, L.; Veenstra, T. D.; Lipton, M. S.; Udseth, H. R.; Smith, R. D. Packed Capillary Reversed-Phase Liquid Chromatography with High-Performance Electrospray Ionization Fourier Transform Ion Cyclotron Resonance Mass Spectrometry for Proteomics. Anal. Chem. 2001, 73, 1766-1775.

15. Kelly, R. T.; Page, J. S.; Luo, Q. Z.; Moore, R. J.; Orton, D. J.; Tang, K. Q.; Smith, R. D. Chemically Etched Open Tubular and Monolithic Emitters for Nanoelectrospray Ionization Mass Spectrometry. Anal. Chem. 2006, $78,7796-7801$.

16. Jaitly, N.; Mayampurath, A.; Littlefield, K.; Adkins, J. N.; Anderson, G. A.; Smith, R. D. Decon2LS: An Open-Source Software Package for Automated Processing and Visualization of High Resolution Mass Spectrometry Data. BMC Bioinformatics 2009, in press.

17. Jaitly, N.; Monroe, M. E.; Petyuk, V. A.; Clauss, T. R. W.; Adkins, J. N.; Smith, R. D. Robust Algorithm for Alignment of Liquid Chromatography-Mass Spectrometry Analyses in an Accurate Mass and Time Tag Data Analysis Pipeline. Anal. Chem. 2006, 78, 7397-7409.

18. Livesay, E. A.; Tang, K. Q.; Taylor, B. K.; Buschbach, M. A.; Hopkins, D. F.; LaMarche, B. L.; Zhao, R.; Shen, Y. F.; Orton, D. J.; Moore, R. J.; Kelly, R. T.; Udseth, H. R.; Smith, R. D. Fully Automated Four-Column Capillary LC-MS System for Maximizing Throughput in Proteomic Analyses. Anal. Chem. 2008, 80, 294-302.

19. Nilsson, S.; Svedberg, M.; Pettersson, J.; Bjorefors, F.; Markides, K.; Nyholm, L. Evaluations of the Stability of Sheathless Electrospray Ionization Mass Spectrometry Emitters Using Electrochemical Techniques. Anal. Chem. 2001, 73, 4607-4616.

20. Chen, M. L.; Cook, K. D. Oxidation Artifacts in the Electrospray Mass Spectrometry of A $\beta$ Peptide. Anal. Chem. 2007, 79, 2031-2036.

21. Geromanos, S.; Freckleton, G.; Tempst, P. Tuning of an Electrospray Ionization Source for Maximum Peptide-Ion Transmission into a Mass Spectrometer. Anal. Chem. 2000, 72, 777-790.

22. Marginean, I.; Kelly, R. T.; Page, J. S.; Tang, K. Q.; Smith, R. D. Toward a Stable Electrospray. In Proceedings of the 55th ASMS Conference on Mass Spectrometry, Indianapolis, IN, June 3-7, 2007.

23. Evans, C. A.; Hendricks, C. D. Electrohydrodynamic Ion-Source for Mass-Spectrometry of Liquids. Rev. Sci. Instrum. 1972, 43, 1527-1530.

24. Chen, D. R.; Pui, D. Y. H.; Kaufman, S. L. Electrospraying of Conducting Liquids for Monodisperse Aerosol Generation in the $4 \mathrm{~nm}$ to $1.8 \mu \mathrm{m}$ Diameter Range. J. Aerosol Sci. 1995, 26, 963-977.

25. Old, W. M.; Meyer-Arendt, K.; Aveline-Wolf, L.; Pierce, K. G.; Mendoza, A.; Sevinsky, J. R.; Resing, K. A.; Ahn, N. G. Comparison of Label-Free Methods for Quantifying Human Proteins by Shotgun Proteomics. Mol. Cell. Proteomics 2005, 4, 1487-1502.

26. Ono, M.; Shitashige, M.; Honda, K.; Isobe, T.; Kuwabara, H.; Matsuzuki, H.; Hirohashi, S.; Yamada, T. Label-Free Quantitative Proteomics Using Large Peptide Data Sets Generated by Nanoflow Liquid Chromatography and Mass Spectrometry. Mol. Cell. Proteomics 2006, 5, 1338-1347.

27. Kelly, R. T.; Page, J. S.; Marginean, I.; Tang, K. Q.; Smith, R. D. Nanoelectrospray Emitter Arrays Providing Interemitter Electric Field Uniformity. Anal. Chem. 2008, 80, 5660-5665. 